\title{
Splitting of Landau levels of a 2D electron due to electron-phonon interactions
}

\author{
V. Cheianov \\ Uppsala University, S-751 08, Uppsala, Sweden \\ A.P. Dmitriev and V.Yu.Kachorovskii \\ A.F.Ioffe Physical-Technical Institute, St.Petersburg, 194021, Russia
}

\begin{abstract}
We show that in a very strong magnetic field $B$ electron-phonon interaction gives rise to a splitting of Landau levels of a 2D electron into a series of infinitely degenerate sublevels. We provide both qualitative and quantitative description of this phenomenon. The cases of interaction with acoustic and polar optical phonons are considered. The energy distance between nearest sublevels in both cases tends to zero as $B^{-1 / 2}$ at large $B$.
\end{abstract}

The dynamics of a 2D lowest Landau level (LL) electron in high mobility samples where only the electronphonon interaction is relevant was disscussed in Refs. 11.2. In the limit of weak electron-phonon coupling the temperature dependence of the longitudinal electron mobility was calculated in these works both at low [1] and relatively high [2] temperatures.

The limiting case of weak electron-phonon interaction is not, however, realized at very high magnetic fields. The effective coupling constant $\alpha$ characterizing the electronphonon interaction in a strong magnetic field increases with $B$ (see below) and may reach large values at which a new physics comes into play due to the essential role of polaronic effects. In this limit the conclusions of Refs. [1.2] are no longer valid. Some aspects of the problem of calculating the electron mobility in this case were discussed in Ref. [3].

In this letter we would like to concentrate on a simpler problem of finding the energy spectrum of the electronphonon system when $B \rightarrow \infty$. Knowledge of this spectrum is nesesary for the correct calculation of the electron mobility at very low temperatures.

For $\alpha \gg 1$ the lattice in the vicinity of an electron is strongly deformed and this deformation creates a polaronic well (PW) around the electron. The characteristic frequency of the electron motion in the PW is very high compared to the phonon frequencies and the lattice deformation forming the $\mathrm{PW}$ can be considered quasistatic. The energy and the wave function of the ground state (GS) of the electron in the PW in the limit $B \rightarrow \infty$ were calculated in Ref. 四 in the framework of Pekar adiabatic method [5]. At first sight it may seem that the results of Ref. [i] give the complete description of the physics of the problem and the polaronic effects simply reduce to an energy shift of the LL. This, in fact, is not true. As we will show, in strong coupling limit the LL splits into a series of sublevels, these sublevels being infinitely degenerate like the original LL.

This splitting can be understood qualitatively on an example of a simple model, which can be solved exactly. Let us consider a $2 \mathrm{D}$ electron with the effective mass $m^{*}$ placed in a strong magnetic field and coupled by a harmonic potential to a heavy neutral particle with the mass $M \gg m^{*}$. This particle models the phonon cloud surrounding an electron in the crystall. There are three different types of motion in the system. The fastest one is the motion of the electron with the cyclotronic frequency $\omega_{c}=e B / m^{*} c$. The second one is a slower drift of the magnetic circle center of the electron around the heavy particle with the frequency $\Omega=\omega_{0}^{2} / \omega_{c}$, where $\omega_{0} \ll \omega_{c}$ is the frequency of the electron oscillations in the harmonic potential in the absence of the magnetic field. The third, slowest type of motion is the cyclotronic rotation of the system as a whole with the frequency $\Omega_{c}=e B / M c$. The energy spectrum of the model can be classified by three quantum numbers $n, m, N$ corresponding to these types of motion $E_{n, m, N}=\hbar \omega_{c} n+\hbar \Omega m+\hbar \Omega_{c} N$.

In case of an electron interacting with phonon field there also exist three types of motion. In analogy with the model, the Landau levels corresponding to the fast cyclotronic rotation of the electron are split due to the slower drift of the magnetic circle center in the PW and to yet slower rotation of the polaron as a whole. In this letter we restrict ourselves to the calculation of the splitting of the lowest LL. As we will show the drift frequency $\Omega$ depends on the radius of the drift orbit and the series associated with the quantum number $m$ is not equidistant. The frequency of the slowest cyclotronic rotation of the polaron as a whole $\Omega_{c}$ is inversely proportional to the polaronic mass $M$, which depends on the magnetic field and the quantum number $m$. The appearance of a finite mass of the polaron (an electron at the LL has effectively no mass), is a result of dressing of the electron by phonons. We want to emphasize that in our considerations the transitions between different LLs of the electron are neglected and the finite polaronic mass arises when the states of one LL only are taken into account. In strong coupling limit the mass $M$ is proportional to $\alpha$ and grows with magnetic field as $B^{3 / 2}$, whereas the distance between nearast sublevels $\hbar \Omega_{c}$ decreases as $B^{-1 / 2}$. Note also, that all the sublevels of the spectrum obtained in our letter are infinitely degenerate over the position of the magnetic circle center of the polaron as a whole.

We consider an electron at zero temperature in a quantum well (QW) of the width $a$ located in the plane $(x, y)$ to which a strong magnetic field $\mathbf{B}$ parallel to the axis 
$z$ is applied. We assume that the electron is at the first level in QW and at the lowest LL. The sample is supposed to be fairly pure and the filling factor of the LL is supposed to be small so that the impurity scattering as well as the electron-electron scattering can be neglected. The Hamiltonian of the system projected on the lowest LL can be written [2, 6] in terms of the operator of magnetic circle center $\mathbf{R}=(\hat{X}, \hat{Y})$ with non-commuting components $[\hat{X}, \hat{Y}]=i l^{2}$ :

$$
H=\sum_{\mathbf{q}} \frac{P_{\mathbf{q}}^{2}+\omega_{\mathbf{q}}^{2} Q_{\mathbf{q}}^{2}}{2}+\sum_{\mathbf{q}} f_{\mathbf{q}} Q_{\mathbf{q}} \sin \left(\mathbf{q}_{||} \mathbf{R}-\pi / 4\right),
$$

where $l$ is the magnetic length, $Q_{\mathbf{q}}$ are the normal coordinates of the oscillations of the lattice and $P_{\mathbf{q}}$ are the momenta conjugated to them, $\omega_{\mathbf{q}}$ is the phonon frequency, $\mathbf{q}$ is the phonon wave vector with the components $\mathbf{q}_{\|}$in the QW's plane and $q_{z}$ in $z$ direction. The function $f_{\mathbf{q}}$ can be written as

$$
f_{\mathbf{q}}=F_{\mathbf{q}} \eta\left(q_{z}\right) e^{-q_{\| \mid}^{2} l^{2} / 4}, \eta\left(q_{z}\right)=\int d z e^{i q_{z} z}\left|\psi_{0}(z)\right|^{2}
$$

The factors $\exp \left(-q_{\|}^{2} l^{2} / 4\right)$ and $\eta\left(q_{z}\right)$ appear as a result of the projecting of the original Hamiltonian of the system on the zero LL and on the lowest level in the QW with the wave function of transversal quantization $\psi_{0}(z)$. Due to these factors the electron effectively interacts with the phonon modes with momenta lying in the shell $q_{\|}<1 / l, q_{z}<1 / a$. The function $F_{\mathbf{q}}$ is proportional to the square root of coupling constant $\alpha$. Its concrete form depends on the type of phonons. We will do all the calculations for arbitrary $F_{\mathbf{q}}$ and afterwards consider two examples of polar optical phonons and acoustic phonons interacting with the electron via deformation potential.

We assume that at $\alpha \gg 1$ characterisic frequency of electron motion in the $\mathrm{PW}$ is much larger than phonon frequencies. Then the PW is quasistatic and one can introduce the wave function $\Psi$ of the GS of the electron in this well. Averaging the Hamiltonian (11) with $\Psi$ we get the Hamiltonian which only depends on the phonon coordinates and momenta. The ground state energy of this Hamiltonian is given by

$$
E\{\Psi\}=-\sum_{\mathbf{q}} \frac{f_{\mathbf{q}}^{2}}{2 \omega_{\mathbf{q}}^{2}}\left\langle\Psi\left|\sin \left(\mathbf{q}_{||} \mathbf{R}-\pi / 4\right)\right| \Psi\right\rangle^{2}
$$

In Pekar method [5] $\Psi$ should be obtained by minimizing the energy functional (3) under the constraint $\langle\Psi \mid \Psi\rangle=1$. One can see that the functional $(\beta)$ is stationary on the eigenfunctions of the angular momentum operator of the electron at the lowest LL $\hat{M}=\hbar\left[l^{2}-\hat{X}^{2}-\hat{Y}^{2}\right] / 2 l^{2}$. This implies that the self consistent potential of the PW is axially symmetric. The absolute minimum of the energy functional (3) is reached on the zero angular momentum wave function [4] which in $X$ representation $\left(\hat{X}=X\right.$ and $\left.\hat{Y}=-i l^{2} d / d X\right)$ has the form
$\Psi_{0}(X)=\left(\pi l^{2}\right)^{-1 / 4} \exp \left(-X^{2} / 2 l^{2}\right)$. The GS energy is given by

$$
E_{0}=-\sum_{\mathbf{q}} \frac{f_{\mathbf{q}}^{2}}{4 \omega_{\mathbf{q}}^{2}} \exp \left(-q_{\|}^{2} l^{2} / 2\right)
$$

Now let us consider the excited polaronic states in the quasiclassical approximation. The Lagrange function corresponding to the Hamiltonian (11) is written as follows

$\mathcal{L}=\frac{\hbar}{l^{2}} \dot{X} Y+\sum_{\mathbf{q}} \frac{\dot{Q}_{\mathbf{q}}^{2}-\omega_{\mathbf{q}}^{2} Q_{\mathbf{q}}^{2}}{2}-\sum_{\mathbf{q}} f_{\mathbf{q}} Q_{\mathbf{q}} \sin \left(\mathbf{q}_{\|} \mathbf{R}-\pi / 4\right)$.

The equations of motion given by this Lagrange function read

$$
\begin{gathered}
\dot{\mathbf{R}}=-\frac{l^{2}}{\hbar} \sum_{\mathbf{q}} f_{\mathbf{q}} Q_{\mathbf{q}}\left[\mathbf{n} \times \mathbf{q}_{\|}\right] \cos \left(\mathbf{q}_{\|} \mathbf{R}-\pi / 4\right), \\
\ddot{Q}_{\mathbf{q}}+\omega_{\mathbf{q}}^{2} Q_{\mathbf{q}}=-f_{\mathbf{q}} \sin \left(\mathbf{q}_{\|} \mathbf{R}-\pi / 4\right),
\end{gathered}
$$

where $\mathbf{n}$ is the unit vector in $z$ direction. Classical motion of the electron in the axially symmetric PW is simply rotation

$$
\mathbf{R}=\mathbf{R}_{\mathbf{0}}+\mathbf{r}=\left(R_{0} \cos (\Omega t)+x, R_{0} \sin (\Omega t)+y\right),
$$

where $\mathbf{r}$ is the position of the PW. After substituting (8) into ( 7 ) we see that the phonon coordinates $Q_{\mathbf{q}}$ can be represented as a sum of harmonics with the frequencies which are integer multiples of $\Omega$. Assuming that $\Omega \gg \omega_{\mathbf{q}}$ we can keep in the sum only the harmonic with zero frequency and obtain the following expression for $Q_{\mathbf{q}}$

$$
Q_{\mathbf{q}} \simeq Q_{\mathbf{q}}^{0}(\mathbf{r})=-\frac{J_{0}\left(q_{||} R_{0}\right) f_{\mathbf{q}}}{\omega_{\mathbf{q}}^{2}} \sin \left(\mathbf{q}_{||} \mathbf{r}-\pi / 4\right) .
$$

Substituting Eqs. (8) and (9) into (6) we get the frequency $\Omega$ of the classical drift of the electron in the effective phonon potential $U\left(R_{0}\right)$

$$
\Omega=\frac{l^{2}}{\hbar R_{0}} \frac{d U\left(R_{0}\right)}{d R_{0}}, \quad U\left(R_{0}\right)=-\sum_{\mathbf{q}} \frac{f_{\mathbf{q}}^{2} J_{0}^{2}\left(q_{||} R_{0}\right)}{4 \omega_{\mathbf{q}}^{2}} .
$$

Quasiclassically the values of the rotation radius $R_{0}$ are quantized as

$$
R_{0 m}=l \sqrt{2 m}, m \gg 1,
$$

where $m$ is an absolute value of the quantum of the angular momentum. In our case $m$ coinsides with the number of the energy level in the PW. The energy of the $m$-th high excited state is given by

$$
E_{m}=U\left(R_{0 m}\right)
$$

These levels are separated by large energy $\sim \hbar \Omega$ proportional to the coupling constant. 
The high frequency motion described above is connected with the internal degrees of freedom of the polaron. Besides these internal degrees of freedom there exist two more degrees of freedom $x, y$ (see Eq.(8)) corresponding to the motion of the polaron as a whole. Now we will show that polaron aquires a finite mass $M \sim \alpha$ and moves as a heavy particle with the electric charge $e$ in magnetic field $B$. Quantization of this motion leads to additional equidistant splitting of energy levels. The energy of splitting is equal to the cyclotronic energy $\hbar \Omega_{c}=\hbar e B / M c$.

First we consider the problem quasiclassically, assuming that the electron in the PW is in one of the highly exited states $(m \gg 1)$. Let us treat vector $\mathbf{r}$ in $(\$)$ as a slowly changing function of time. Then the deformation field $Q_{q}^{0}$ will adiabatically follow the motion of the polaron according to Eq. (9). Let us do the following change of variables $Q_{q}=Q_{q}^{0}(\mathbf{r})+\theta_{\mathbf{q}}$, where $\theta_{q}$ stands for the oscillations of the phonon coordinates around $Q_{q}^{0}$. Substituting Eqs.(8) into (5) and averaging over the fast rotation with the ferquency $\Omega$ we get the effective Lagrange function corresponding to the slow motion of the polaron

$$
\mathcal{L}_{\mathrm{ef}}=\frac{\hbar}{l^{2}} y \dot{x}+\frac{M_{m} \dot{\mathbf{r}}^{2}}{2}+\sum_{\mathbf{q}} \frac{\dot{\theta}_{\mathbf{q}}^{2}-\omega_{\mathbf{q}}^{2} \theta_{\mathbf{q}}^{2}}{2}+\mathcal{L}_{\mathrm{int}}
$$

The first two terms in Lagrange function (13) describe the motion of a massive particle with the electric charge $e$ in the magnetic field $B$. Classically this motion is cyclotronic rotation with the frequency $\Omega_{c}$. The mass $M_{m}$ of the polaron in $m$-th exited state is given by

$$
M_{m}=\sum_{\mathbf{q}} \frac{f_{\mathbf{q}}^{2} J_{0}^{2}\left(q_{\|} R_{0 m}\right)}{4 \omega_{q}^{4}} q_{\|}^{2}
$$

The term $\mathcal{L}_{\text {int }}$ in the Lagrange function describes the interaction of polaron with the phonons $\theta_{\mathbf{q}}$. This interaction leads to decay of the cyclotronic rotation of the polaron and $\Omega_{c}$ acquires imaginary part $\Gamma$. For acoustic phonons $\Gamma$ is small as compared to $\Omega_{c}$ if the velocity of the cyclotronic motion is less than the sound velocity. For optical phonons the decay appears as long as we take into account the dispersion of phonon frequency. For weak dispersion $\Gamma$ is also small. In both cases the quantization of cyclotronic rotation of the polaron leads to well defined Landau levels of the polaron as a whole.

Quasiclassical approximation is not valid if we consider the cyclotronic splitting of the GS. Still in this case it is also possible to derive the effective Lagrange function for the slow motion of the polaron. This can be done using the standard procedure of integrating out fast components $\mathbf{R}_{\mathbf{0}}(t)$ of the vector $\mathbf{R}(t)$ in functional integral describing transition amplitudes of the electron interacting with the phonon field. The resulting effective Lagrange function will only differ from (13) by concrete form of the interaction term and by the expression for the mass $M$. Namely, in Eq. (14) the Bessel function $\mathrm{J}_{0}\left(q_{\|} R_{0 m}\right)$, which had appeared as a result of classical averaging over fast rotation should be replaced by the quantum average of $\exp \left(i \mathbf{q}_{\|} \mathbf{R}\right)$ over the GS of the electron $\exp \left(i y X / l^{2}\right) \Psi_{0}(X-x)$ in the PW placed at the point $(x, y)$. For the mass of the polaron in the GS we have

$$
M_{0}=\sum_{\mathbf{q}} \frac{f_{\mathbf{q}}^{2} q_{\|}^{2}}{4 \omega_{q}^{4}} \exp \left(-\frac{q_{\|}^{2} l^{2}}{2}\right) .
$$

The highly exited states of an electron in the PW can decay into lower exited states, the energy difference being transfered to the polaron as a whole. The rigorous calculation of corresponding transition probability is cumbersome. One can argue, however, that this probability is negligibly small. Indeed, the momentum $P$ transfered to polaron in this process is $P=\sqrt{2 M\left(E_{m}-E_{m^{\prime}}\right)} \sim$ $\sqrt{M \hbar \Omega} \sim \alpha$. In strong coupling limit $P$ is large compared to $\hbar / l$ and cannot be compensated by phonon emission due to the factor $\exp \left(-q_{\|}^{2} l^{2} / 4\right)$ in Eq.(2/2).

The equations obtained above are general and can be applied to any type of electron-phonon interaction. Next we consider two concrete examples of an electron interacting with acoustic and polar optical phonons. For certainity we will assume that $a \ll l$.

1. Acoustic phonons. For acoustic phonons

$$
F_{q}=\sqrt{\frac{2 \pi \alpha \hbar l^{2} s \omega_{q}^{2}}{V}}, \quad \alpha=\frac{C_{0}^{2}}{\pi \rho \hbar l^{2} s^{3}} .
$$

Here $\alpha \sim B$ is the dimensionless coupling constant characterizing the electron-phonon interaction in a strong magnetic field , $C_{0}$ is the deformation potential constant, $\rho$ is the density of the crystal, $s$ is the sound velosity, and $V$ is the volume of the crystall.

Performing the summation in Eqs.(4) we obtain energy of the GS of the polaron

$$
E_{0}=-\frac{\alpha \hbar \omega_{a}}{8 \pi}, \omega_{a}=\frac{s}{2} \int_{-\infty}^{\infty} d q_{z} \eta^{2}\left(q_{z}\right) \simeq \frac{s}{a}
$$

Classical frequency of rotation of the electron in the PW is determined by Eq.(10). Taking into account that in Eq.(10) $R_{0} \gg l$ we obtain the following expression for the drift frequency

$$
\Omega=\frac{\alpha \omega_{a}}{\pi \sqrt{32 \pi}}\left(\frac{l}{R_{0}}\right)^{3}
$$

Eq. (10) was derived under the assumption that $\Omega \gg \omega_{q}$. The phonon frequencies are restricted by $\omega_{a}$. Taking into account Eq. (11) we have the following condition of the validity of our calculations

$$
\alpha \gg 1, \quad m \ll \alpha^{2 / 3} \text {. }
$$

By the virtue of (10) and (12) for the energy levels in quasiclassical approximation we have 


$$
E_{m}=-\frac{\alpha \hbar \omega_{a}}{8 \pi^{3 / 2} \sqrt{m}}
$$

The mass of the polaron in ground and highly excited states are obtained from Eqs. (14, 15)

$$
M_{0}=\alpha \frac{\sqrt{\pi} \hbar}{32 l s}, M_{m}=\alpha \frac{\hbar}{8 \pi l s \sqrt{2 m}} .
$$

The spacing between Landau levels of the polaron depends on $m$ and is equal to $\hbar \Omega_{c}^{0}=\hbar e B /\left(M_{0} c\right)$ and $\hbar \Omega_{c}^{m}=\hbar e B /\left(M_{m} c\right)$ for ground and highly exited states correspondingly. Viewing the term $\mathcal{L}_{\text {int }}$ in Eq.(13) as a perturbation one can get the following result for the imaginary parts $\Gamma_{0}$ and $\Gamma_{m}$ of the cyclotronic frequencies $\Omega_{c}^{0}$ and $\Omega_{c}^{m}$

$$
\frac{\Gamma_{0}}{\Omega_{c}^{0}}=\frac{8 \pi}{3}\left(\frac{32}{\pi \alpha}\right)^{3}, \frac{\Gamma_{m}}{\Omega_{c}^{m}}=\frac{8 \pi m^{2}}{3}\left(\frac{8 \pi}{\alpha}\right)^{3}
$$

to the first order in small parameter $1 / \alpha^{3}$. We see that in a strong magnetic field when $\alpha$ is large $\Gamma$ is small compared to the spacing $\Omega_{c}$ and Landau levels of the polaron are well defined. Eqs.(22) are valid if the polaron velosity is smaller than the sound velosity. In the opposite case $\Gamma$ becomes very large and cyclotronic quantization of polaron motion is absent.

2. Optical phonons. For optical phonons

$$
F_{q}=\sqrt{\frac{8 \alpha l \hbar \omega_{0}^{3}}{q^{2} V}}, \quad \alpha=\frac{\pi e^{2}}{\hbar \omega_{0} l}\left(\frac{1}{\epsilon_{\infty}}-\frac{1}{\epsilon_{0}}\right) .
$$

Here $\alpha \sim \sqrt{B}$ is the dimensionless coupling constant for optical phonons in a strong magnetic field and $\omega_{0}$ is the optical phonon frequency . The expressions for the drift frequency and the energy of an electron and the mass of the polaron in ground and highly exited states read

$$
\begin{gathered}
\Omega=\frac{\alpha \omega_{0}}{2 \pi^{2}} \frac{l^{3} \ln \left(R_{0} / l\right)}{R_{0}^{3}}, \\
E_{0}=-\frac{\alpha \hbar \omega_{0}}{\sqrt{8 \pi}}, \quad E_{m}=-\frac{\alpha \hbar \omega_{0}}{4 \pi^{2}} \frac{\ln m}{\sqrt{2 m}} . \\
M_{0}=\frac{\alpha \hbar}{8 \sqrt{\pi} \omega_{0} l^{2}}, \quad M_{m}=\frac{\alpha \hbar}{\pi^{2} \omega_{0} l^{2} \sqrt{8 m}} .
\end{gathered}
$$

It follows from Eqs.(21,26) that for given $m$

$$
M \sim B^{3 / 2}, \quad \Omega_{c} \sim B^{-1 / 2} .
$$

The above results are in contrast with the usual point of view [7] that the motion of the electron strongly interacting with phonons in a magnetic field is characterized by two frequencies only: the frequency of the fast motion of the electron in the PW and the frequency of the cyclotronic rotation of the polaron as a whole with the mass not depending (or slightly depending) on $B$. In fact this is true only when $\omega_{c}$ is small compared with the characteristic frequency of the electron motion in the PW. Our results are valid in the opposite case.

Note finally, that the splitting of the LL can be apparently observed in Ge samples, where the deformation potential is considerably large. Simple estimation shows that in this material effective coupling constant $\alpha$ becomes sufficiently large at $B>50 \mathrm{~T}$.

\section{ASCKNOWLEGEMENTS}

The authors are grateful to A.Alekseev, A.Barybin, and M.Dyakonov for very useful discussions. This work was supported in part by the Swedish Royal Academy of Science (grant 1240) and by the Russian Foundation for Basic Research (Grants 96-02-17894 a and 96-02-17896). Partial support for one of the authors (V.Yu.K.) was provided by a fellowship of INTAS Grant 93-2492-e within the research program of International Center for Fundamental Physics in Moscow. This work was also supported by Grant 1001 within the program "Physics of Solid State Nanostructures".

[1] Yu.A.Bychkov, S.V.Iordanski, and G.M.Eliashberg, Pis'ma v Zh.Eksp. Teor.Fiz 34, 496, (1981).[Sov.Phys.JETP Lett.34, 496, 1981]

[2] A.P.Dmitriev and V.Yu.Kachorovskii, Physical Review B 52, pp.5743-5751, (1995).

[3] L.S.Kukushkin, D.G.Radchenko, and A.V.Frentiy Physics of Low Temperatures 20, pp. 448-452, (1994)

[4] E.D.Vol and L.S.Kukushkin, Physics of Low Temperatures ,9, 97-99, (1983). L.S.Kukushkin, Pis'ma v Zh.Eksp.Teor.Fiz., 7, pp. 251-253, (1968)

[5] S.I.Pekar, Zh.Eksp. Teor.Fiz17, 868, (1947)

[6] Note, that here we write the Hamiltonian in the "stationary wave" representation which is different from the one used in Ref.( 2|

[7] "Polarons in Ionic Crystals and Polar Semiconductors", Ed.Jozef T.Devreese, North-Holland Publishing Company, Amsterdam, London, (1972) 